\title{
Allergy-Preventive Effects of the Flowers of Impatiens textori
}

\author{
Emiko Iwaoka, ${ }^{a}$ Hisae OKu, ${ }^{b}$ Munekazu Innum, ${ }^{c}$ and Kyoko Ishiguro $*, b$ \\ ${ }^{a}$ Department of Pharmacy, Hyogo University of Health Sciences; Minatojima, Chuo-ku, Kobe 650-8530, Japan: ${ }^{b}$ School \\ of Pharmacy and Pharmaceutical Sciences, Mukogawa Women's University; Koshien Kyuban-cho, Nishinomiya, Hyogo \\ 663-8179, Japan: and ${ }^{c}$ Laboratory of Pharmacognosy, Gifu Pharmaceutical University; 5-6-1 Mitahora-higashi, Gifu \\ 502-8585, Japan. Received December 8, 2009; accepted December 21, 2009; published online January 25, 2010
}

The allergy-preventive activity of a 35\% EtOH extract (IT) of flowers of Impatiens textori MiQ. was demonstrated in a continuing search for allergy-preventive substances from natural sources. The evaluation of its activity used an in vivo assay method for monitoring the blood flow decrease in the tail vein microcirculation of mice subjected to sensitization with hen-egg white lysozyme. Among the principal compounds in IT, apigenin (1), luteolin (3), and luteolin 7-glucoside (4) showed significant allergy-preventive effects.

Key words Impatiens textori; allergy-preventive effect; blood flow; luteolin; apigenin; hen-egg white lysozyme

Whole plant of Impatiens textori MiQ. (Balsaminaceae) is an annual herbaceous plant that grows naturally along waterfronts in the mountains in almost every region of Japan. It has been used for detoxication and treatment of carbuncles and contusions in Chinese medicine. ${ }^{1)}$ However, there has been no report on the antiallergic effects of the flowers of I. textori. In a previous study, we demonstrated the antianaphylactic, antipruritic, and antiplatelet activating factor (PAF) activities of a 35\% EtOH extract (IT) of the flowers of I. textori, and isolated apigenin (1), apigenin 7-glucoside (2), luteolin (3), luteolin 7-glucoside (4), quercetin (5), kaempferol (6), and kaempferol 3-glucoside (7).,3)

In a continuing search for allergy-preventive substances from natural sources, using our previously developed in vivo assay method to estimate complicated allergy, ${ }^{4}$ we found that IT exhibited allergy-preventive activity. This in vivo assay method monitors the decrease in blood flow in the tail vein of mice subjected to hen egg-white lysozyme (HEL) sensitization alone without the HEL challenge as a guide. ${ }^{4)}$ The blood flow in HEL-sensitized mice (control group) gradually and significantly decreased to about $70 \%$ of that in normal mice on day 9. Thus, the induction phase of allergy caused by xenobiotics can be dynamically and easily measured using blood flow monitoring. This blood flow decrease is considered to be due to the contraction of peripheral blood vessels and increase in blood viscosity, because no relationship with blood pressure was observed. Although anti-HEL immunoglobulin E (IgE) antibody significantly increased after HEL sensitization, there was no significant increase in the number of leukocytes. Thus the decrease in blood flow reflects the promoter process of an allergic reaction. The blood flow decrease is regulated by various factors such as nitric oxide (NO), thromboxane (TX) $\mathrm{A}_{2}$, prostacyclin $\left(\mathrm{PGI}_{2}\right)$, and endothelin (ET)-1, together with granulocytic elastase (GE), cyclooxygenase (COX)-1 and -2 , inducible nitric oxide synthase (iNOS), and constitutive nitric oxide synthase (cNOS). ${ }^{4)}$ Additionally, the blood flow decrease occurs via both pathways of the iNOS-independent and -dependent response. ${ }^{5)}$ Therefore this monitoring system should be useful for searching for preventive substances against complicated allergy involving NO, TXA 2 , PGI 2 , ET-1, GE, COX-1, and COX-2.,5)

This paper describes the evaluation of the allergy-preventive effects of a $35 \%$ EtOH extract (IT) of the flowers of $I$. textori and compounds $(\mathbf{1}-\mathbf{7})$ previously isolated from IT.

\section{MATERIALS AND METHODS}

Materials The $35 \%$ EtOH extract (IT) of the flowers of I. textori was used for our present and previous studies. ${ }^{2,3)}$ Apigenin (1), apigenin 7-glucoside (2), luteolin (3), luteolin 7-glucoside (4), quercetin (5), kaempferol (6), and kaempferol 3-glucoside (7) were isolated from IT as previously reported. ${ }^{2)}$ HEL and complete Freund's adjuvant (CFA) were purchased from Sigma Chemical (St. Louis, MO, U.S.A.), Difco (Detroit, Michigan, U.S.A.), respectively.

Animals Male ddY mice (SPF grade), 5 weeks old, were obtained from Japan SLC, Inc. (Shizuoka, Japan) and housed at $24 \pm 2{ }^{\circ} \mathrm{C}$. Food and water were available ad libitum. All experiments were performed in accordance with the Guidelines for Animal Experiments of Mukogawa Women's University.

HEL Sensitization Immunization with HEL was performed as previously described. ${ }^{4}$ Male ddY mice, 5 weeks of age, were sensitized intraperitoneally with $50 \mu \mathrm{g}$ of HEL in $50 \%$ CFA on day 0 .

Blood Flow Measurement Subcutaneous blood flow in the tail vein of the mice was monitored using a laser Doppler blood flow meter of the noncontact type (FLO-C1, Neuro-

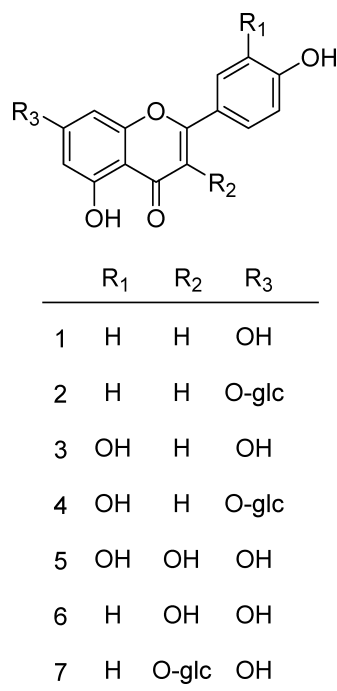

(C) 2010 Pharmaceutical Society of Japan 
science, Tokyo, Japan), as previously reported. ${ }^{4)}$ Each mouse was prewarmed for $10 \mathrm{~min}$ at $36^{\circ} \mathrm{C}$ prior to the experiment and was placed on a holder in a measuring chamber kept at $36^{\circ} \mathrm{C}$ throughout the measurement. The blood flow was measured for $10 \mathrm{~min}$ without anesthesia. The results are expressed as mean \pm S.E. $(n=5$ or 7$)$ of the percentage of the normal blood flow of each mouse, measured $1 \mathrm{~d}$ before experiment due to large individual differences in blood flow.

Assay of Allergy-Preventive Activity IT $(200 \mathrm{mg} / \mathrm{kg})$ and each isolated compound $(20 \mathrm{mg} / \mathrm{kg})$ were dissolved in water and administered ( $10 \mu \mathrm{l}$ of test solution/g body weight) orally on days 0 (the starting day; $1 \mathrm{~h}$ before sensitization), 3 , 6 , and 9. The statistical calculations were determined in comparison with the HEL-sensitized mice (control group).

Statistical Analysis Statistical analysis was performed using Dunnett's multiple-range test coupled with Bonferroni inequality for significant differences between each test group and the control group. For the Bonferroni test, 5 points were used after day 4 of the HEL sensitization, because a significance difference was observed between the blood flow of untreated and sensitized mice after day 4 .

\section{RESULTS}

The blood flow of HEL-sensitized mice (control group) gradually and significantly $(p<0.05)$ decreased to about $50 \%$ of the blood flow of normal mice on day 9 (Fig. 1). On the other hand, the oral administration of IT significantly $(p<0.05)$ improved the HEL-induced blood flow decrease, which reflects the promoter process of an allergic reaction, compared with the control group after day 5 of HEL sensitization, as shown in Fig. 1. Neither IT nor its compounds affected the blood flow after oral administration in normal mice without HEL sensitization. Thus IT could be useful for preventing complicated allergy development.

Apigenin (1) significantly improved the HEL-induced blood flow decrease compared with that in the control group after day 9 of HEL sensitization, as shown in Fig. 2A. Apigenin 7-glucoside (2), which is a glucoside of apigenin, show a tendency to improve the blood flow decrease in HEL-sensitized ddY mice (Fig. 2A). Luteolin (3) and luteolin 7-glucoside (4) also significantly $(p<0.05)$ improved the HEL-induced blood flow decrease compared with that in the control group after day 5 of HEL sensitization (Fig. 2B). Quercetin (5), kaempferol (6), and kaempferol 3-glucoside (7) did not significantly inhibit the blood flow decrease in HEL-sensitized ddY mice, as shown in Figs. 2C and D. Compounds $1-$ 7 alone did not affect the blood flow. Therefore compounds 1-4 may play important roles in the allergy-preventive activity of IT.

\section{DISCUSSION}

The present data confirm the allergy-preventive activity of IT, since it significantly improved the blood flow decrease, which reflects the promoter process of an allergic reaction by HEL sensitization. Among the constituents of IT, apigenin (1), apigenin 7-glucoside (2), luteolin (3), and luteolin 7-glucoside (4) may play important roles in the allergy-preventive activity.

Our previous paper reported that the mechanisms of HEL-

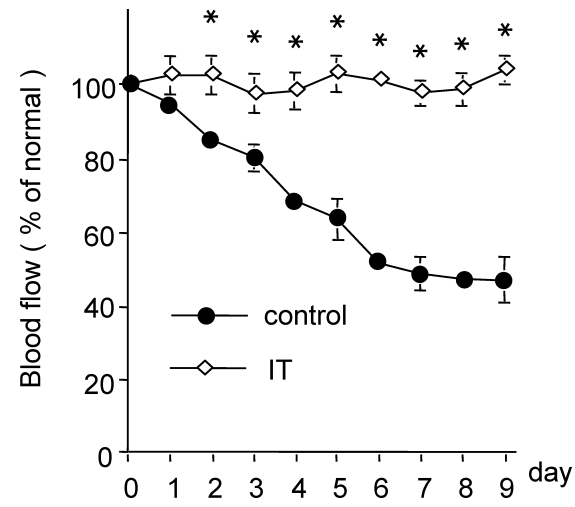

Fig. 1. Allergy-Preventive Effects of the $35 \%$ EtOH Extract (IT) of the Flowers of I. textori

- Control (blood flow of HEL-sensitized mice); $\diamond$, pretreatment with IT $(200 \mathrm{mg} / \mathrm{kg}$, p.o. $)$, on days 0 ( $1 \mathrm{~h}$ before sensitization $), 3,6$, and 9 after sensitization. Each value presents the mean \pm S.E. for 7 mice. $* p<0.05$ compared with the control group (Dunnett's test with Bonferroni)
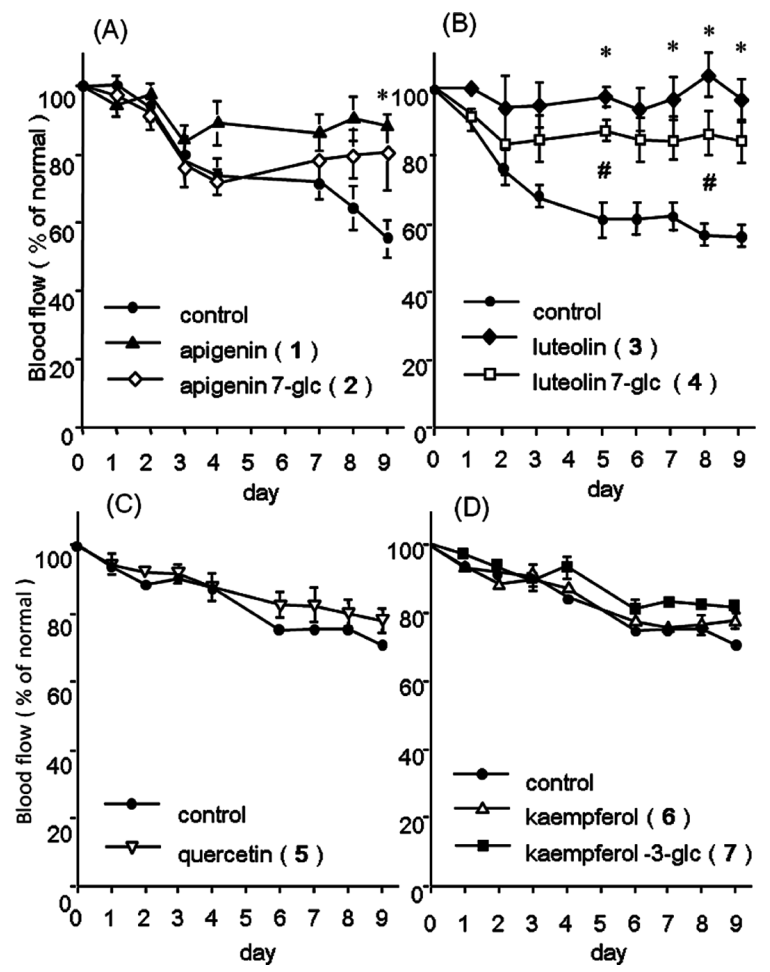

Fig. 2. Allergy-Preventive Effects of the Principal Compounds from IT

- Control (blood flow of HEL-sensitized mice), (A); $\boldsymbol{\Lambda}$, pretreatment with apigenin (1); $\diamond$, pretreatment with apigenin 7-glucoside (2), (B); $\diamond$, pretreatment with luteolin (3); $\square$, pretreatment with luteolin 7-glucoside (4); (C) $\nabla$, pretreatment with quercetin (5), (D); $\triangle$, pretreatment with kaempferol (6); $\mathbf{\square}$, pretreatment with kaempferol 3-glucoside (7). The compounds $(20 \mathrm{mg} / \mathrm{kg}$, p.o.) were administered on days 0 ( $1 \mathrm{~h}$ before sensitization), 3, 6, and 9 after sensitization. Each value presents the mean \pm S.E. for 5 mice. $* p<0.05$ compared with the control group (Dunnett's test with the Bonferroni).

induced blood flow decrease are complicated and involve various factors such as $\mathrm{NO}, \mathrm{PGI}_{2}, \mathrm{TXA}_{2}, \mathrm{ET}-1, \mathrm{COX}-1$, COX-2, cNOS, iNOS, and GE. ${ }^{4,5)}$ The blood flow is maintained at a normal level until HEL sensitization, because NO from eNOS regulates vessel tonus. After sensitization, cytokines induced by HEL activate neutrophilic leukocytes that release GE, and injury of vascular endothelial cells by GE inhibits the production of $\mathrm{NO}$ and $\mathrm{PGI}_{2}$. As a result, the relatively predominant ET-1 induces microcirculation failure. Furthermore, the decrease in $\mathrm{NO}$ and $\mathrm{PGI}_{2}$ and the increase 
in $\mathrm{TXA}_{2}$ promote platelet aggregation, followed by blood flow decrease. The resulting ischemia facilitates further production of cytokines, which induces the expression of COX-2 and iNOS, induces enhancement of the immune response, and leads to a vicious cycle of blood flow decrease. During this process, iNOS expression is not indispensable, but perhaps assists in amplifying the serious decrease in blood flow.

The biological activities of apigenin (1) and luteolin (3) have been reported, such as antagonism of the $\mathrm{TXA}_{2}$ receptor $^{6,7)}$ and inhibitory effects on the signaling pathway from the $\mathrm{TXA}_{2}$ receptor, ${ }^{6}$ iNOS and COX-2 expression, ${ }^{8}$ NO production, and platelet aggregation in vitro. ${ }^{9)}$ In addition, compound $\mathbf{3}$ has inhibitory effects on the Toll-like receptor (TLR) signaling pathway. ${ }^{10)}$

Thus it is assumed that inhibitory mechanisms of IT on the HEL-induced blood flow decrease are wide and variable. Compounds $\mathbf{1}$ and $\mathbf{3}$ inhibit platelet aggregation, because they inhibit binding of the $\mathrm{TXA}_{2}$ receptor and signaling pathway from the $\mathrm{TXA}_{2}$ receptor. ${ }^{6,7)}$ The compounds inhibit the expression of iNOS and COX-2 induced by HEL sensitization. ${ }^{8}$ Compound 3 inhibits the TLR signaling pathway, which plays important roles in the release of cytokines and mediators from activated macrophages, neutrophilic cells, acidophilic cells, and vascular endothelial cells, followed by activation of the immune response. As a result, compound 3 attenuates vascular endothelial cell injury and vascular hyperpermeability. ${ }^{10)}$ Therefore IT inhibits the formation of microthrombus and finally improves microcirculatory disturbances. Details of the mode of action of IT and its principal compounds are under investigation using this animal model.

To our knowledge, this is the first report of the allergy-preventive effects of IT using an animal model to evaluate the allergy induction phase (before allergy development). We previously reported the inhibitory effects of IT on established allergy such as antianaphylactic, antipruritic, and anti-PAF activity. ${ }^{2,3)}$ Thus this flower could be useful during the preventive and therapeutic stages of allergy.

\section{REFERENCES}

1) Chang S., "Dictionary of Chinese Crude Drugs," New Medical College Shanghai Scientific Technological Publishers, Shanghai, 1977.

2) Ueda Y., Oku H., Iinuma M., Ishiguro K., Biol. Pharm. Bull., 28, 1786-1790 (2005)

3) Ueda Y., Oku H., Iinuma M., Ishiguro K., Biol. Pharm. Bull., 26, 1505-1507 (2003)

4) Ishiguro K., Oku H., Ueda Y., Iwaoka E., Kunitomo M., Biol. Pharm. Bull., 28, 1490-1495 (2005).

5) Oku H., Ogawa Y., Iwaoka E., Kunitomo M., Ueda H., Okamura H., Ishiguro K., Biol. Pharm. Bull., 30, 1324-1328 (2007).

6) Guerrero J. A., Navarro-Nuñez L., Lozano M. L., Martínez C., Vicente V., Gibbins J. M., Rivera J., Br. J. Clin. Pharmacol., 64, 133-144 (2007).

7) Guerrero J. A., Lozano M. L., Castillo J., Benavente-García O., Vicente V., Rivera J., J. Thromb. Haemost., 3, 369-376 (2005).

8) Lopez-Pasadas R., Ballester I., Abadia-Molina A. C., Suarez M. D., Zarzuelo A., Martinez-Augustin O., Sanchez de Medina F., Biochem. Pharmacol., 76, 495-506 (2008).

9) Lin C. N., Kuo S. H., Chung M. I., J. Nat. Prod., 60, 851-853 (1997).

10) Lee J. K., Kim S. Y., Kim Y. S., Lee W.-H., Hwang D. H., Lee J. Y., Biochem. Pharmacol., 77, 1391-1400 (2009). 